\title{
A NOTE ON THE LOCAL INVERTIBILITY OF SOBOLEV FUNCTIONS
}

\author{
ROBERTO VAN DER PUTTEN
}

\begin{abstract}
.
We give some topological and analytical conditions in order that a continuous Sobolev function be a local homeomorphism. The results are obtained in the setting of the spaces $W^{1, n}\left(\Omega ; \mathrm{R}^{n}\right)$ and $W^{2, p}\left(\Omega ; \mathbf{R}^{n}\right)$.
\end{abstract}

\section{Introduction.}

In this paper we deal with the local invertibility of continuous mappings and, more precisely, with the properties of the branch set of such mappings; we recall that, if $\Omega$ is an open subset of $\mathbf{R}^{n}$ and $f: \Omega \rightarrow \mathbf{R}^{n}$ a continuous mapping, the branch set of $f$, denoted by $B_{f}$, is the set of all points $x \in \Omega$ where $f$ does not define a local homeomorphism. It is well known that if $f \in C^{1}$, then $B_{f} \subset Z_{f}$ where $Z_{f}=\{x \in \Omega: D f(x)$ exists and $\operatorname{det} D f(x)=0\}$, but the study of $B_{f}$ becomes more difficult beyonds the class of smooth mappings. Some results have been obtained under topological assumptions: if $f$ is light and sense-preserving (see below for definitions) then the topological dimension of $B_{f}$ and $f\left(B_{f}\right)$ is not greater than $n-2$ and

$$
B_{f} \subset Z_{f} \cup S_{f}
$$

where $S_{f}=\{x \in \Omega: f$ is not weakly differentiable at $x\}$ ([11] and [3]).

However, it is not known under what analytical conditions a mapping is light and sense-preserving; some results can be found in [7] (mappings with finite dilatation) and in the monograph of Rickman ([11]) on quasiregular mappings.

Invertibility has been studied also in the setting of nonlinear elasticity: in fact this requirement guarantees that interpenetration of matter does not occur. In this case Ball and Šverak ([2], [13]) have found analytical conditions which implies the global invertibility of Sobolev functions.

Received March 11, 1996. 
In this paper we present three results in the setting of Sobolev spaces: the first two concern mappings belonging to $W^{1, n}\left(\Omega ; \mathrm{R}^{n}\right)$ and they are slight improvements of the recalled result in [11] (Chap. I, Lemma 4.11); we prove that (1.1) holds if $\operatorname{det} D f \geq 0$ almost everywhere in $\Omega$ and $f$ is either open or light. The topological degree is widely used in the proofs. The third theorem concerns mappings belonging to $W^{2, p}\left(\Omega ; \mathrm{R}^{n}\right)$. First we prove that $S_{f}$ is a set of zero capacity if $p>\frac{n(n-1)}{2 n-1}$; then we use this result to show that (1.1) holds if $p>n-1, Z_{f}$ is a set of zero capacity and $\operatorname{det} D f>0$ almost everywhere in $\Omega$.

\section{Notations and preliminaries.}

Throughout this paper $\Omega$ is a nonempty, bounded and open set in $\mathrm{R}^{n}$, with $n \geq 2$.

We write $\mathscr{L}^{n}$ for the Lebesgue measure in $\mathrm{R}^{n}$ and \|\| for the norm in the same space. Given $x \in \mathrm{R}^{n}$ and $r>0, B(x, r)$ is the open ball of center $x$ and radius $r ; Q(x, r)$ is the set $\left\{y \in \mathrm{R}^{n}:\left|x_{i}-y_{i}\right|<r, i \in\{1, \ldots, n\}\right\}$, where $x=$ $\left(x_{1}, \ldots, x_{n}\right)$ and $y=\left(y_{1}, \ldots, y_{n}\right)$. If $A \subset \mathrm{R}^{n}, D(A)$ will be the set of accumulation points of $A$.

For $1 \leq p \leq+\infty$ and $m \geq 1$, let $L^{p}\left(\Omega ; \mathrm{R}^{m}\right)$ be the collection of all $m$-tuples $\left(f_{1}, \ldots, f_{m}\right)$ of real functions in $L^{p}(\Omega)$. For $k \geq 1$, we say that $f \in W^{k, p}\left(\Omega ; \mathbf{R}^{m}\right)$ if $f \in L^{p}\left(\Omega ; \mathrm{R}^{m}\right)$ together with its derivatives (in the sense of distribution) up to $k$ th order; $D f$ will be the distributional Jacobian matrix of $f$.

Now we introduce the Bessel capacity. Let $g$ be the Bessel kernel, that is the function whose Fourier transform is

$$
(\breve{g})(x)=(2 \pi)^{-\frac{n}{2}}\left(1+\|x\|^{2}\right)^{-\frac{1}{2}} ;
$$

for $p>1$, we define the Bessel capacity for any set $A \subset \mathrm{R}^{n}$ as

$$
B_{1, p}(A)=\inf \left\{\int_{\mathrm{R}^{n}}|f(x)|^{p} d x: f \in L^{p}\left(\mathrm{R}^{n}\right), g * f \geq 1 \text { on } A, f \geq 0\right\},
$$

where $g * f$ is the convolution of $g$ and $f$ (the elementary properties of Bessel capacity can be found in [15]).

Let $A \subset \mathrm{R}^{n}$. The Hausdorff dimension of $A$ is defined by $\operatorname{dim}_{H}(A)=\sup \left\{\alpha \geq 0: H^{\alpha}(A)>0\right\}$ with the convention $\operatorname{dim}_{H}(\emptyset)=0$, where $H^{\alpha}$ is the $\alpha$-dimensional Hausdorff measure (see [4]).

Now let $f: \Omega \rightarrow \mathbf{R}^{n}$ be a continuous mapping. We say that $f$ satisfies the condition $(N)$ on $\Omega$ if $\mathscr{L}^{n}(f(A))=0$ whenever $A \subset \Omega$ is such that $\mathscr{L}^{n}(A)=0$. If $A \subset \Omega$ and $y \in \mathrm{R}^{n}$, we denote by $N(f, A, y)$ the number (possibly infinite) of elements of the set $A \cap f^{-1}(y)$. The map $f$ is said to be light if $f^{-1}(y)$ is totally disconnected for every $y \in \mathrm{R}^{n}$. 
In the following $A$ will be a domain such that $A \subset \subset \Omega$ and $y \in \mathrm{R}^{n}$.

Now we introduce the topological degree. Suppose that $y \notin f(\partial A)$. Then there exists $r \in \mathrm{R}, 0<r<1, r$ small enough, such that $f$ induces a homomorphism of cohomology groups

$$
\left.f^{*}: H_{n+1} \overline{\left(B\left(y, r^{-1}\right)\right.} ; \overline{B\left(y, r^{-1}\right)} \backslash B(y, r)\right) \rightarrow H_{n+1}(\bar{A} ; \partial A) .
$$

If $g_{1}, g_{2}$ are suitable generators of the cohomology groups, there exists an integer, we denote it $\mu(y, f, A)$, such that $f^{*}\left(g_{1}\right)=\mu(y, f, A) g_{2} ; \mu(y, f, A)$ is called the topological degree of $y$ with respect to the pair $(f, A)$.

We say that $f$ is sense-preserving (weakly sense-preserving) if $\mu(y, f, A)>$ $0(\mu(y, f, A) \geq 0)$ for every domain $A \subset \subset \Omega$ and $y \notin f(\partial A)$.

Now, resorting to the topological degree, we may define some multiplicity functions. Given a domain $B$ such that $\bar{B} \subset A$, we say that $B$ is a positive (negative) indicator domain for $(y, f, A)$ if $y \notin f(\partial B)$ and $\mu(y, f, B)>0(<0)$. A finite (possibly empty) collection of pair-wise disjoint positive (negative) indicator domains for $(y, f, A)$ is called a positive (negative) indicator system for $(y, f, A)$ and is denoted by $\sigma^{+}(y, f, A)\left(\sigma^{-}(y, f, A)\right)$. Finally we define the multiplicity functions

$$
\begin{array}{r}
K^{+}(y, f, A)=\operatorname{Sup}\left\{\sum_{B \in \sigma^{+}} \mu(y, f, B): \sigma^{+}=\sigma^{+}(y, f, A)\right\}, \\
K^{-}(y, f, A)=\operatorname{Sup}\left\{-\sum_{B \in \sigma^{-}} \mu(y, f, B): \sigma^{-}=\sigma^{-}(y, f, A)\right\}, \\
K(y, f, A)=K^{+}(y, f, A)+K^{-}(y, f, A)
\end{array}
$$

with the convention that $K^{+}(y, f, A)=0\left(K^{-}(y, f, A)=0\right)$ if there is no positive (negative) nonempty indicator system. The multiplicity function $K$ is related with the concept of essential maximal model continua (e.m.m.c.). We say that $C \subset \mathrm{R}^{n}$ is an e.m.m.c. for $(y, f, A)$ if $C$ is a component of $A \cap f^{-1}(y)$ which is a continuum and if for every open set $D$ such that $C \subset D \subset A$ there exists a positive or negative indicator domain $B$ for $(y, f, A)$ such that $C \subset B \subset \bar{B} \subset D$. If either $K(y, f, A) \leq 1$ or $K(y, f, A)=+\infty$ then $K(y, f, A)$ agrees with the number of e.m.m.c. for $(y, f, A)$ ([10], II.3.4., Thm. 3).

A sequence $\left\{B_{k}\right\}_{k \in \mathrm{N}}$ of nonempty domains is called a determining sequence for $(y, f, A)$ if $\overline{B_{k+1}} \subset B_{k} \subset \subset A, y \in f\left(\overline{B_{k}}\right) \backslash f\left(\partial B_{k}\right)$, for every $k \in \mathrm{N}$ and $\lim _{k \rightarrow \infty} \operatorname{diam}\left(f\left(\overline{B_{k}}\right)\right)=0$.

Now let $x_{0} \in \Omega$; we say that $f$ has a weak differential at $x_{0}$ if there exists a linear mapping $L: \mathrm{R}^{n} \rightarrow \mathrm{R}^{n}$ and a set $B \subset \mathrm{R}$ such that 0 is a point of right density of $B$ satisfying 


$$
\lim _{\substack{t \rightarrow 0^{+} \\ t \in B}} \sup \left\{\left\|\frac{f\left(x_{0}+t z\right)-f\left(x_{0}\right)}{t}-L(z)\right\|: z \in \partial Q(0,1)\right\}=0 .
$$

The following result by Goffman and Ziemer ([6] ,Thm. 3.4) states the weak differentiability properties of Sobolev functions.

THEOREM 2.1. If $f \in W^{1, p}\left(\Omega ; \mathrm{R}^{n}\right)$ with $p>n-1$, then $f$ is weakly differentiable almost everywhere in $\Omega$.

\section{Local invertibility of Sobolev functions.}

THEOREM 3.1. Let $f \in W^{1, n}\left(\Omega ; \mathbf{R}^{n}\right)$ be a continuous, open mapping such that $\operatorname{det} D f \geq 0$ almost everywhere in $\Omega$. Then $B_{f} \subset Z_{f} \cup S_{f}$.

Proof. Let $x_{0} \notin Z_{f} \cup S_{f}$; we shall prove that $x_{0} \notin B_{f}$. We use the link between the weak differential and the topological degree ([10], p. 329); if $x_{0} \notin Z_{f} \cup S_{f}$, there exist $r_{1}, r_{2}>0$ such that $Q\left(x_{0}, r_{1}\right) \subset \subset \Omega$ and $\mu\left(y, f, Q\left(x_{0}, r_{1}\right)\right)=1$ for every $y \in B\left(f\left(x_{0}\right), r_{2}\right)$.

We show that $N\left(y, f, Q\left(x_{0}, r_{1}\right)\right)=1$ for every $y \in B\left(f\left(x_{0}\right), r_{2}\right)$. Since $f$ is weakly sense-preserving ([11], Ch. VI, Lemma 5.1), we have $\sigma^{-}\left(y, f, Q\left(x_{0}, r_{1}\right)\right)=\emptyset$ for every $y \in \mathrm{R}^{n}$; therefore $K^{-}\left(y, f, Q\left(x_{0}, r_{1}\right)\right)=0$ and $K\left(y, f, Q\left(x_{0}, r_{1}\right)\right)=K^{+}\left(y, f, Q\left(x_{0}, r_{1}\right)\right)$ for every $y \in \mathbf{R}^{n}$. Furthermore

$$
K^{+}\left(y, f, Q\left(x_{0}, r_{1}\right)\right)=\mu\left(y, f, Q\left(x_{0}, r_{1}\right)\right)=1
$$

for every $y \in B\left(f\left(x_{0}\right), r_{2}\right)$ such that $K\left(y, f, Q\left(x_{0}, r_{1}\right)\right)<+\infty$ ([10], II. 3.4, Thm. 2 and Thm. 4). Now we note that $f$ satisfies the condition (N) ([8], Corollary B) and, by Theorem $2.1, f$ is weakly differentiable almost everywhere in $\Omega$. This implies that $K\left(\cdot, f, Q\left(x_{0}, r_{1}\right)\right) \in L^{1}\left(\mathrm{R}^{n}\right)$ ([10], V. 3.3., Thm. 5) and then $K\left(y, f, Q\left(x_{0}, r_{1}\right)\right)<+\infty$ for almost every $y \in \mathrm{R}^{n}$. By (3.1), $K\left(y, f, Q\left(x_{0}, r_{1}\right)\right)=1$ for almost every $y \in B\left(f\left(x_{0}\right), r_{2}\right)$ and, therefore, also $N\left(y, f, Q\left(x_{0}, r_{1}\right)\right)=1$ for almost every $y \in B\left(f\left(x_{0}\right), r_{2}\right)$ ([10], V. 3.3. Thm. 2). Since $f$ is open, then $N\left(\cdot, f, Q\left(x_{0}, r_{1}\right)\right)$ is lower semicontinuous in $\mathrm{R}^{n}$ ([5], Chap. 5, Thm. 1.3) and this implies that $N\left(\cdot, f, Q\left(x_{0}, r_{1}\right)\right) \leq 1$ everywhere in $B\left(f\left(x_{0}\right), r_{2}\right)$. On the other hand $B\left(f\left(x_{0}\right), r_{2}\right) \subset f\left(Q\left(x_{0}, r_{1}\right)\right)$ because $\mu\left(y, f, Q\left(x_{0}, r_{1}\right)\right)=1$ for every $y \in B\left(f\left(x_{0}\right), r_{2}\right)$ and then $N\left(\cdot, f, Q\left(x_{0}, r_{1}\right)\right)=1$ everywhere in $B\left(f\left(x_{0}\right), r_{2}\right)$.

Finally, let $A_{x_{0}}=f^{-1}\left(B\left(f\left(x_{0}\right), r_{2}\right)\right) \cap Q\left(x_{0}, r_{1}\right)$; since $f\left(A_{x_{0}}\right) \subset B\left(f\left(x_{0}\right), r_{2}\right)$, the restriction of $f$ to $A_{x_{0}}$ is one-to-one and, as $f$ is an open mapping, $f$ is a homeomorphism from $A_{x_{0}}$ to $f\left(A_{x_{0}}\right)$, that is $x_{0} \notin B_{f}$.

Definition 3.2. Let $f: \Omega \rightarrow \mathbf{R}^{n}$ be a continuous mapping. For every $y \in \mathrm{R}^{n}$ we define $R_{y}=\left\{x \in f^{-1}(y)\right.$ : there exists a sequence $\left\{V_{m}\right\}_{m \in \mathrm{N}}$ of open 
neighbourhood of $x$ such that $\operatorname{diam}\left(V_{m}\right)<\frac{1}{m}$, there exists $\mu\left(y, f, V_{m}\right)$ and it is positive for every $m \in N\}$.

Remark 3.3. If $f$ is light, weakly sense-preserving and $A \subset \Omega$ is a domain, we have that $R_{y} \cap A$ is the set of the e.m.m.c. for $(y, f, A)$.

In the following lemma, we recall a property of the set $R_{y}$ for such mappings (see also [14]).

Lemma 3.4. Let $f: \Omega \rightarrow \mathbf{R}^{n}$ be a continuous, light and weakly sense-preserving mapping. Then $D\left(R_{y}\right) \cap \Omega=\emptyset$ for every $y \in \mathrm{R}^{n}$.

Proof. By contradiction we suppose that there exists $y \in \mathrm{R}^{n}$ such that $D\left(R_{y}\right) \cap \Omega \neq \emptyset$. Then let $x \in \Omega$ be a limit point of a sequence $\left\{x_{m}\right\}_{m \in \mathrm{N}}$ in $R_{y}$. Since $f$ is continuous and light, then $x \in f^{-1}(y)$ and there exists an open neighbourhood $B$ of $x$ such that $B \subset \Omega$ and $f^{-1}(y) \cap \partial B=\emptyset$. Let $\mu(y, f, B)=\alpha \geq 0, k \in \mathrm{N}$ satisfying $k>\alpha$ and let $\left\{\bar{x}_{1}, \bar{x}_{2}, \ldots . ., \bar{x}_{k}\right\}$ be $k$ points of the sequence $\left\{x_{m}\right\}_{m \in \mathrm{N}}$ belonging to $B$. Then, for every $i \in\{1,2, \ldots, k\}$, there exists an open neighbourhood $V_{i}$ of $\bar{x}_{i}$ such that $\bar{V}_{i} \subset B, \bar{V}_{j} \cap \bar{V}_{l}=\emptyset$ for every $j, l \in\{1,2, \ldots, k\}$ and $\mu\left(y, f, V_{i}\right) \geq 1$.

Finally let $\quad V=\cup_{i=1}^{k} V_{i}$ and $\Omega=B \backslash \bar{V}$. Then $f^{-1}(y) \cap \partial V=\emptyset$, $f^{-1}(y) \cap \partial W=\emptyset$ and therefore $f^{-1}(y) \cap B \subset \cup_{i=1}^{K} V_{i} \cup W$. Consequently

$$
\alpha=\mu(y, f, B)=\sum_{i=1}^{K} \mu\left(y, f, V_{i}\right)+\mu(y, f, W) \geq k
$$

and this is a contradiction.

THeOREM 3.5. Let $f \in W^{1, n}\left(\Omega ; \mathbf{R}^{n}\right)$ be a continuous, light mapping such that $\operatorname{det} D f \geq 0$ almost everywhere in $\Omega$. Then $B_{f} \subset Z_{f} \cup S_{f}$.

Proof. As in Theorem 3.1, we consider $x_{0} \notin Z_{f} \cup S_{f}$ and we prove that $x_{0} \notin B_{f}$. Analogously we can show that there exist $r_{1}, r_{2}>0$ such that $K\left(y, f, Q\left(x_{0}, r_{1}\right)\right)=1$ for every $y \in B\left(f\left(x_{0}\right), r_{2}\right)$ satisfying $K\left(y, f, Q\left(x_{0}, r_{1}\right)\right)<$ $+\infty$.

Now we prove that $\left\{y \in B\left(f\left(x_{0}\right), r_{2}\right): K\left(y, f, Q\left(x_{0}, r_{1}\right)\right)=+\infty\right\}=\emptyset$. By contradiction we suppose that there exists $y \in B\left(f\left(x_{0}\right), r_{2}\right)$ such that $K\left(y, f, Q\left(x_{0}, r_{1}\right)\right)=+\emptyset$. Then let $\left\{C_{i}\right\}_{i \in \mathrm{N}}$ be a sequence of e.m.m.c. for $\left(y, f, Q\left(x_{0}, r_{1}\right)\right)$; since $f$ is light, we have $C_{i}=\left\{x_{i}\right\}$ for every $i \in \mathrm{N}$, and $x_{i} \in R_{y}$ for every $i \in \mathrm{N}$. Therefore $R_{y} \cap Q\left(x_{0}, r_{1}\right)$ contains a bounded, infinite subset and this implies that $D\left(R_{y}\right) \cap \Omega \neq \emptyset$, which contradicts Lemma 3.4. Hence we have $K\left(y, f, Q\left(x_{0}, r_{1}\right)\right)=1$ for every $y \in B\left(f\left(x_{0}\right), r_{2}\right)$ and, by Remark 3.3, this implies that $R_{y} \cap Q\left(x_{0}, r_{1}\right)=\left\{x_{y}\right\}$ for every $y \in B\left(f\left(x_{0}\right), r_{2}\right)$.

Now let $g(y)=x_{y}$ for every $y \in B\left(f\left(x_{0}\right), r_{2}\right)$. We prove that $g$ is a homeomorphism and $g=f^{-1}$ in a neighbourhood of $f\left(x_{0}\right)$. 
First we show that $g$ is continuous on $B\left(f\left(x_{0}\right), r_{2}\right)$. Let $y \in B\left(f\left(x_{0}\right), r_{2}\right)$ be a limit point of a sequence $\left\{y_{m}\right\}_{m \in \mathrm{N}}$ in $B\left(f\left(x_{0}\right), r_{2}\right)$ and suppose for contradiction that there exists a subsequence $\left\{x_{y_{m_{k}}}\right\}_{k \in \mathrm{N}}$ which converges to $x \in \overline{Q\left(x_{0}, r_{1}\right)}$ and $x \neq x_{y}$. We have that $x \in f^{-1}(y)$ and therefore $x \in Q\left(x_{0}, r_{1}\right)$. Hence $x \notin R_{y}$ and, since $f$ is light, this implies that there exists an open neighbourhood $W$ of $x$ such that $\mu(y, f, W)=0$; consequently, there exists $h \in \mathrm{N}$ such that $\mu\left(y_{m_{k}}, f, W\right)=0$ for every $k \geq h$. On the other hand there exists an open neighbourhood $V_{m}$ of $x_{y_{m}}$ such that $\operatorname{diam}\left(V_{m}\right)<\frac{1}{m}$ and $\mu\left(y, f, V_{m}\right) \geq 1$; then, if we take $h^{*} \in \mathrm{N}$ such that $h^{*} \geq h$ and $V_{m_{k}} \subset \subset W$ for every $k \geq h^{*}$ we have

$$
\mu\left(y_{m_{k}}, f, W\right)=\mu\left(y_{m_{k}}, f, V_{m_{k}}\right)+\mu\left(y_{m_{k}}, f, W \backslash \bar{V}_{m_{k}}\right)=0
$$

for every $k \geq h^{*}$. Hence $\mu\left(y_{m_{k}}, f, V_{m_{k}}\right)=0$ and this is a contradiction. Therefore $g$ is continuous on $B\left(f\left(x_{0}\right), r_{2}\right)$.

Now we observe that, by the definition of $R_{y}, f(g(y))=y$ for every $y \in B\left(f\left(x_{0}\right), r_{2}\right)$ and then $g$ is one-to-one. Therefore, by the invariance domain theorem, $g$ is open and then it is a homeomorphism from $B\left(f\left(x_{0}\right), r_{2}\right)$ to $g\left(B\left(f\left(x_{0}\right), r_{2}\right)\right)$; consequently $f=g^{-1}$ is a homeomorphism from $g\left(B\left(f\left(x_{0}\right), r_{2}\right)\right)$ to $B\left(f\left(x_{0}\right), r_{2}\right)$. Finally we observe that $g\left(f\left(x_{0}\right)\right)=x_{0}$ ([10], pag 329) and $g\left(B\left(f\left(x_{0}\right), r_{2}\right)\right)$ is an open neighbourhood of $x_{0}$. Therefore $x_{0} \notin B_{f}$.

REMARK 3.6. Theorems 3.1 and 3.5 are not true without the topological assumptions about $f$, i.e. if $f$ is neither open nor light, as it is shown by the following example : let $k \in \mathrm{N}, I=(-2,2), I_{k}=\left(k^{-1}, k^{-1}+k^{-2}\right), F=\cup_{k \in N} I_{k}$ and $E=F \cup(-F)$; it is easily verified that $x_{0}=0$ is a point of density 1 for $E$ (see [4] for the definition). We set

$$
g(x)=\int_{0}^{x} \chi_{E}(t) d t \text { and } f(x, y)=(g(x), y) \text { if }(x, y) \in I \times I
$$

where $\chi_{E}$ is the characteristic function of $E$. Then $f \in W^{1, \infty}\left(I \times I ; \mathrm{R}^{2}\right)$ and $\operatorname{det} D f(x, y)=\chi_{E}(x) \geq 0$ for almost every $(x, y) \in I \times I$.

Now we observe that $(0,0) \in B_{f} \backslash\left(Z_{f} \cup S_{f}\right)$. Indeed, if $k \in \mathrm{N}$, let $J_{k}=\left([k+1]^{-1}+[k+1]^{-2}, k^{-1}\right)$; we note that $g^{\prime}(x)=0$ for every $x \in \cup_{k \in \mathrm{N}} J_{k}$ and therefore $g$ is constant on each $J_{k}$. Then, for every $\delta>0$ we may choose $m \in \mathrm{N}$ such that $J_{m} \subset(-\delta ; \delta)$ and $f\left(J_{m} \times\{0\}\right)$ is a point. This implies that $(0,0) \in B_{f}$. On the other hand, since $x_{0}=0$ is a point of density 1 for $E$, we have that $f$ is weakly differentiable in $(0,0)$ and $\operatorname{det} D f(0,0)=1$, that is $(0,0) \notin\left(Z_{f} \cup S_{f}\right)$. Finally, we note that $\mathrm{f}$ is neither open nor light ; indeed $f\left(J_{k} \times I\right)=\left\{g\left(k^{-1}\right)\right\} \times I$ and $f^{-1}\left(g\left(k^{-1}\right), 0\right)=\bar{J}_{k} \times\{0\}$ for every $k \in \mathrm{N}$. 


\section{Local invertibility of Sobolev functions of higher order.}

The following lemma concerns a local property of the fibers $f^{-1}(y)$; the proof is similar to the one of Theorem 1, (iv) in [2].

LEMMA 4.1. Let $f \in W^{1, n}\left(\Omega ; \mathrm{R}^{n}\right)$ be a continuous mapping such that $\operatorname{det} D f>0$ almost everywhere in $\Omega$ and let $x_{0} \notin Z_{f} \cup S_{f}$.

Then there exist $r_{1}, r_{2}>0$ such that $f^{-1}(y) \cap Q\left(x_{0}, r_{1}\right)$ is a continuum for every $y \in B\left(f\left(x_{0}\right), r_{2}\right)$.

Proof. Since $x_{0} \notin Z_{f} \cup S_{f}$, there exist $r_{1}, r_{2}>0$ such that $Q\left(x_{0}, r_{1}\right) \subset \subset \Omega$ and $\mu\left(y, f, Q\left(x_{0}, r_{1}\right)\right)=1$ for every $y \in B\left(f\left(x_{0}\right), r_{2}\right)$ (see the proof of Theorem $3.1)$.

First we observe that $f^{-1}(y) \cap Q\left(x_{0}, r_{1}\right)$ is closed. In fact for every $y \in B\left(f\left(x_{0}\right), r_{2}\right), f^{-1}(y) \cap \partial Q\left(x_{0}, r_{1}\right)$ is empty and since $Q\left(x_{0}, r_{1}\right) \subset \subset \Omega$ and $f^{-1}(y)$ is closed in $\Omega$, we have $\overline{f^{-1}(y) \cap Q\left(x_{0}, r_{1}\right)} \subset Q\left(x_{0}, r_{1}\right)$ and this implies that $f^{-1}(y) \cap Q\left(x_{0}, r_{1}\right)$ is closed.

Now let's suppose by contradiction that $f^{-1}(y) \cap Q\left(x_{0}, r_{1}\right)$ is not connected and consider a partition of compact sets $\left\{C_{i}\right\}_{i=1,2}$ of $f^{-1}(y) \cap Q\left(x_{0}, r_{1}\right)$; besides let $A_{1}, A_{2}$ be disjoint open sets such that $C_{i} \subset A_{i} \subset Q\left(x_{0}, r_{1}\right)(i=1,2)$. Since $y \notin f\left(\partial A_{1}\right) \cup f\left(\partial A_{2}\right)$ we have

$$
1=\mu\left(y, f, Q\left(x_{0}, r_{1}\right)\right)=\mu\left(y, f, A_{1}\right)+\mu\left(y, f, A_{2}\right) .
$$

Without loss of generality we can suppose $\mu\left(y, f, A_{1}\right) \leq 0$. Then there exists $\alpha>0$ such that $B(y, \alpha) \cap\left[f\left(\partial A_{1}\right) \cup f\left(\partial A_{2}\right)\right]$ is empty and $\mu\left(z, f, A_{1}\right) \leq 0$ for every $z \in B(y, \alpha)$; now, $f$ satisfies condition $(N)$ on $\Omega$ ([9], Corollary 3.13) and therefore, as in Theorem 3.1, one can prove that $K\left(y, f, A_{1}\right)<+\infty$ for almost every $y \in \mathrm{R}^{n}$. Hence the following transformation formula holds ([10], II.3.4., Thm 2 and V.3.4., Thm 1)

$$
\int_{A_{1}} \chi_{B(y, \alpha)}(f(x)) \operatorname{det} D f(x) d x=\int_{B(y, \alpha)} \mu\left(z, f, A_{1}\right) d z
$$

and consequently $\mathscr{L}^{n}\left(A_{1} \cap f^{-1}(B(y, \alpha))\right)=0$. Since $A_{1} \cap f^{-1}(B(y, \alpha))$ is open, it follows that $A_{1} \cap f^{-1}(B(y, \alpha))=\emptyset$ and therefore $C_{1} \cap f^{-1}(y)=\emptyset$, which is a contradiction.

In the following we suppose that $\Omega$ has a Lipschitz boundary (see [1]).

LEMMA 4.2. If $f \in W^{2, p}\left(\Omega ; \mathrm{R}^{n}\right)$ with $p>\frac{n(n-1)}{2 n-1}$, then $B_{1, p}\left(S_{f}\right)=0$.

Proof. From the Sobolev inequalities it follows that $f \in W^{1, p}\left(\Omega ; \mathbf{R}^{n}\right)$ with $p>n-1$ and then, according to the proof of Theorem 3.4 in [6], we have 


$$
\begin{aligned}
\Omega \backslash S_{f} \supset & \left\{x \in \Omega : D f ( x ) \text { exists and } \operatorname { l i m } _ { t \rightarrow 0 ^ { + } } \left[t^{-n} \int_{Q(x ; 2 t)}\|D f(y)-D f(x)\|^{p} d y+\right.\right. \\
& \left.\left.+t^{-n-p} \int_{Q(x ; 2 t)}\|f(y)-f(x)-D f(x)(y-x)\|^{p} d y\right]=0\right\} .
\end{aligned}
$$

Since $D f \in W^{1, p}\left(\Omega ; \mathrm{R}^{n^{2}}\right)$, there exists a set $E \subset \Omega$ such that $B_{1, p}(E)=0$ and

$$
\lim _{t \rightarrow 0^{+}} t^{-n} \int_{Q(x ; 2 t)}\|D f(y)-D f(x)\|^{p} d y=0
$$

for every $x \in \Omega \backslash E$ ([15], Theorem 3.3.3.). Besides, there exists a set $F \subset \Omega$ such that $B_{1, p}(F)=0$ and

$$
\lim _{t \rightarrow 0^{+}} t^{-n-p} \int_{Q(x ; 2 t)}\|f(y)-f(x)-D f(x)(y-x)\|^{p} d y=0
$$

for every $x \in \Omega \backslash F$ ([15], Theorem 3.4.2.). Finally, if we define $G=E \cup F$, we obtain $S_{f} \subset G$ and $B_{1, p}(G)=0$.

In the next theorem we deal with mappings $f$ belonging to $W^{2, p}\left(\Omega ; \mathbf{R}^{n}\right)$ with $p>n-1$. We recall that, by the Sobolev inequalities, such mappings have a Hölder continuous representative in their equivalence class; we shall always assume that this representative of $\mathrm{f}$ has been choosen.

THEOREM 4.3. Let $p>n-1$ and $f \in W^{2, p}\left(\Omega ; \mathrm{R}^{n}\right)$ such that

(a) $\operatorname{det} D f>0$ almost everywhere in $\Omega$,

(b) $B_{1, p}\left(Z_{f}\right)=0$.

Then $B_{f} \subset Z_{f} \cup S_{f}, \operatorname{dim}_{H}\left(B_{f}\right) \leq n-p$ and $\operatorname{dim}_{H}\left(f\left(B_{f}\right)\right) \leq \frac{p(n-p)}{2 p-n}$.

Proof. Let $x_{0} \notin Z_{f} \cup S_{f}$; we shall prove that $x_{0} \notin B_{f}$. First we observe that, by the Sobolev inequalities, $f \in W^{1, n}\left(\Omega ; \mathbf{R}^{n}\right)$ and satisfies the condition $(N)$ ([9], Corollary 3.13); as in the proof of Theorem 3.1, we can show that there exist $r_{1}, r_{2}>0$ and a set $N \subset B\left(f\left(x_{0}\right), r_{2}\right)$ such that $\mathscr{L}^{n}(N)=0$ and $N\left(y, f, Q\left(x_{0}, r_{1}\right)\right)=1$ for every $y \in B\left(f\left(x_{0}\right), r_{2}\right) \backslash N$.

Let $A_{x_{0}}=f^{-1}\left(B\left(f\left(x_{0}\right), r_{2}\right)\right) \cap Q\left(x_{0}, r_{1}\right)$ and let's prove that $N\left(y, f, Q\left(x_{0}, r_{1}\right)\right)=1$ for every $y \in f\left(A_{x_{0}}\right)$. For this purpose we show that $f^{-1}(y) \cap Q\left(x_{0}, r_{1}\right) \subset\left(Z_{f} \cup S_{f}\right) \cap A_{x_{0}}$ for every $y \in N \cap f\left(A_{x_{0}}\right)$.

Let $y \in N \cap f\left(A_{x_{0}}\right)$ and $x \in f^{-1}(y) \cap Q\left(x_{0}, r_{1}\right)$.

First we prove that $x \in\left(\Omega \backslash A_{x_{0}}\right) \cup\left(Z_{f} \cup S_{f}\right)$. Let's suppose by contra- 
diction that $x \in A_{x_{0}} \backslash\left(Z_{f} \cup S_{f}\right)$. By the Hölder continuity of $f$, there exists a sequence of positive real numbers $\left\{\alpha_{n}\right\}_{n \in \mathrm{N}}$ such that $\left\{Q\left(x ; \alpha_{n}\right)\right\}_{n \in \mathrm{N}}$ is a determining sequence for $\left(y, f, Q\left(x_{0}, r_{1}\right)\right)([10]$, p.329). This implies that $\{x\}$ is a component of $f^{-1}(y) \cap Q\left(x_{0}, r_{1}\right) \quad$ ([10], II.3.1., Lemma 6); besides $y \in B\left(f\left(x_{0}\right), r_{2}\right)$ and therefore $f^{-1}(y) \cap Q\left(x_{0}, r_{1}\right)$ is a continuum by Lemma 4.1; consequently $\{x\}=f^{-1}(y) \cap Q\left(x_{0}, r_{1}\right)$ and $y \notin N$, which is a contradiction. Then $x \in\left(\Omega \backslash A_{x_{0}}\right) \cup\left(Z_{f} \cup S_{f}\right)$.

Finally we show that $x \in A_{x_{0}}$. Again by contradiction, let's suppose $x \notin A_{x_{0}}$. Since $x \in Q\left(x_{0}, r_{1}\right)$, we have $x \notin f^{-1}\left(B\left(f\left(x_{0}\right), r_{2}\right)\right)$ and this implies that $x \notin f^{-1}(y)$, otherwise $f(x)=y \in f\left(A_{x_{0}}\right) \subset B\left(f\left(x_{0}\right), r_{2}\right)$, and this is a contradiction. Then $x \in A_{x_{0}} \cap\left[\left(\Omega \backslash A_{x_{0}}\right) \cup\left(Z_{f} \cup S_{f}\right)\right]=A_{x_{0}} \cap\left(Z_{f} \cup S_{f}\right)$ and consequently $f^{-1}(y) \cap Q\left(x_{0}, r_{1}\right) \subset\left(Z_{f} \cup S_{f}\right) \cap A_{x_{0}}$ for every $y \in N \cap f\left(A_{x_{0}}\right)$.

Now, by Lemma 4.2 and (b), we obtain

$$
B_{1, p}\left(f^{-1}(y) \cap Q\left(x_{0}, r_{1}\right)\right) \leq B_{1, p}\left(Z_{f} \cup S_{f}\right)=0
$$

for every $y \in N \cap f\left(A_{x_{0}}\right)$. Since $p>n-1$, we have

$$
\operatorname{dim}_{H}\left(f^{-1}(y) \cap Q\left(x_{0}, r_{1}\right)\right)<1
$$

([15], Th. 2.6.16), and, since $f^{-1}(y) \cap Q\left(x_{0}, r_{1}\right)$ is a continuum by Lemma 4.1, then $\operatorname{diam}\left(f^{-1}(y) \cap Q\left(x_{0}, r_{1}\right)\right)=0$, that is $N\left(y, f, Q\left(x_{0}, r_{1}\right)\right)=1$. Therefore we have proved that $N\left(y, f, Q\left(x_{0}, r_{1}\right)\right)=1$ for every $y \in N \cap f\left(A_{x_{0}}\right)$ and then for every $y \in f\left(A_{x_{0}}\right)$. This implies that the restriction of $f$ to $A_{x_{0}}$ is one - to one; by the invariance domain theorem it follows that such restriction is open and then $f$ is a homeomorfism from $A_{x_{0}}$ to $f\left(A_{x_{0}}\right)$, that is $x_{0} \notin B_{f}$.

In order to show that $\operatorname{dim}_{H}\left(B_{f}\right) \leq n-p$, it is enough to recall that, by (b) and Lemma 4.2, $B_{1, p}\left(B_{f}\right) \leq B_{1, p}\left(Z_{f}\right)+B_{1, p}\left(S_{f}\right)=0$. Hence $H^{n-p+\epsilon}\left(B_{f}\right)=0$ for every $\epsilon>0$ ([15], Theorem 2.6.16) and $\operatorname{dim}_{H}\left(B_{f}\right) \leq n-p$.

Finally we observe that, by the Sobolev inequalities, $f \in C^{o,(2 p-n) / p}\left(\Omega ; \mathrm{R}^{n}\right)$ and therefore $H^{p(n-p+\epsilon) /(2 p-n)}\left(f\left(B_{f}\right)\right) \leq H^{n-p+\epsilon}\left(B_{f}\right)=0$ for every $\epsilon>0$ ([12], Theorem 29); consequently $\operatorname{dim}_{H}\left(f\left(B_{f}\right)\right) \leq \frac{p(n-p)}{2 p-n}$.

\section{REFERENCES}

1. R. Adams, Sobolev spaces, Academic Press, New York, 1975.

2. J. M. Ball, Global invertibility of Sobolev functions and the interpenetration of matter, Proc. Royal Soc. Edinburgh 88 A (1981), 315-328.

3. P. T. Church and E. Henningsen, Light open maps on n-manifolds, Duke Math. J. 27 (1960), 527-536.

4. L. C. Evans and R. F. Gariepy, Measure Theory and Fine Properties of Functions, CRC Press, 1992.

5. V. M. Gol'dshtein and Yu. G. Reshetnyak, Quasiconformal mappings and Sobolev spaces, Kluwer Academic publishers, Dordrecht, 1990. 
6. C. Goffman and W. P. Ziemer, Higher dimensional mappings for which the area formula holds, Ann. of Math. (2) 92 (1970), 482-488.

7. J. Heinonen and P. Koskela, Sobolev mappings with integrable dilatation, Arch. Rational Mech. Anal. 125 (1993), 81-97.

8. J. Maly and O. Martio, Lusin's condition $(N)$ and mappings of the class $W^{1, n}$, J. Reine Angew. Math. 458 (1995), 19-36.

9. O. Martio and W. P. Ziemer, Lusin's condition $(N)$ and mappings with non-negative Jacobians, Michigan Math. J. 39 (1992), 495-508.

10. T. Radó and P. V. Reichelderfer, Continuous transformation in analysis, Springer-Verlag, Berlin, 1955.

11. S. Rickman, Quasiregular Mappings, Springer-Verlag, Berlin, 1993.

12. C. A. Rogers, Hausdorff Measures, Cambridge University Press, 1970.

13. V. Šverak, Regularity properties of deformations with finite energy, Arch. Rat. Mech. Anal. 100 (1988), 105-127.

14. Ju. Ju. Trohimčuk, Continuous mappings of domains in euclidean space, Ukrain. Mat. Ž. 16 (1964), 196-211; English transl., Amer. Math. Soc. Transl. (2) 100 (1972), 271-291.

15. W. P. Ziemer, Weakly Differentiable Functions, Springer-Verlag, Berlin, 1989.

DIPARTIMENTO DI MATEMATICA

VIA DODECANESO, 35

16146 GENOVA

ITALIA 